\title{
Entanglement production in a chaotic quantum dot
} Beenakker, C.W.J.; Kindermann, M.; Marcus, C.M.; Yacoby, A.

\section{Citation}

Beenakker, C. W. J., Kindermann, M., Marcus, C. M., \& Yacoby, A. (2004). Entanglement production in a chaotic quantum dot. In . Kluwer. Retrieved from https://hdl.handle.net/1887/1298

Version: $\quad$ Not Applicable (or Unknown)

License: $\quad$ Leiden University Non-exclusive license

Downloaded from: $\quad$ https://hdl.handle.net/1887/1298

Note: To cite this publication please use the final published version (if applicable). 


\title{
Chapter 10
}

\section{ENTANGLEMENT PRODUCTION IN A CHAOTIC QUANTUM DOT}

\author{
C.W.J. Beenakker, M. Kindermann \\ Instituut-Lorentz, Universitett Leiden PO Box 95062300 RA Letden The Netherlands
}

C.M. Marcus, A. Yacoby*

Department of Physıcs, Harvard Untversity, Cambr ldge, MA 02138, USA

\begin{abstract}
It has recently been shown theoretically that elastic scattering in the Fermi sea produces quantum mechanically entangled states The mechanism is similai to entanglement by a beam splitter in optics, but a key distinction is that the electronic mechanism works even if the source is in local thermal equilibirum An exper1mental realization was proposed using tunneling between two edge channels in a stiong magnetic held Here we investigate a low magnetic field alternative, using multuple scattering in a quantum dot Two pairs of single-channel point contacts define a pail of qubits If the scatteing is chaotic, a universal statistical description of the entanglement production (quantified by the concurrence) is possible The mean concurience turns out to be almost independent on whether time reversal symmetry is bioken or not We show how the concurence can be extracted fiom a Bell inequality using low-trequency noise measurements, without requiring the tunneling assumption of earliei work
\end{abstract}

Keywords: entanglement, Bell mequality, quantum chaos, quantum dot

\section{Introduction}

The usual methods for entanglement production rely on interactions between the particles and the resulting nonlinearities of their dynamics. A text book example fiom optics is parametric down-conversion, which produces a

\footnotetext{
*Visiting fiom Depattment of Condensed Mattei Physıcs, Weizmann Institute of Science Rehovot 76100, Isiael
} 
polarization-entangled Bell pair at frequency $\omega$ out of a single photon at frequency $2 \omega$ [1]. In condensed matter the schemes proposed to entangle electrons make use of the Coulomb interaction or the superconducting pairing interaction [2].

Photons can be entangled by means of linear optics, using a beam splitter, but not if the photon source is in a state of thermal equilibrium [3-5]. Remarkably enough, this optical "no-go theorem" does not carry over to electrons: It was discovered recently [6] that single-particle elastic scattering can create entanglement in an electron reservoir even if it is in local thermal equilibrium. The existence of a Fermi sea permits for electrons what is disallowed for photons. The possibility to entangle electrons without interactions opens up a range of applications in solid-state quantum information processing [7-10].

Any two-channel conductor containing a localized scatterer can be used to entangle the outgoing states to the left and right of the scatterer. The particular implementation described in Ref. [6] uses tunneling between edge channels in the integer quantum Hall effect. In this contribution we analyze an alternative implementation, using scattering between point contacts in a quantum dot. We then need to go beyond the tunneling assumption of Ref. [6], since the transmission eigenvalues $T_{1}, T_{2}$ through the quantum dot need not be $\ll 1$.

The multiple scattering in the quantum dot allows for a statistical treatment of the entanglement production, using the methods of quantum chaos and randommatrix theory [11-13]. The interplay of quantum chaos and quantum entanglement has been studied extensively in recent years [14-20], in the context of entanglement production by interactions. The interaction-free mechanism studied here is a new development.

The geometry considered is shown in Fig. 10.1. A quantum dot is connected at the left and at the right to an electron reservoir. The connection is via point contacts connected to single-channel leads. (Spin degeneracy of the channels is disregarded for simplicity.) There are two leads at the left $(\mathrm{L} 1, \mathrm{~L} 2)$ and two leads at the right (R1, R2). A current is passed through the quantum dot in response to a voltage difference $V$ between the two reservoirs. We consider the entanglement between the left and right channels in the energy range $\mathrm{eV}$ above the Fermi energy $E_{F}$.

The degree of entanglement is measured through the violation of a Bell inequality [21] for correlators of current fluctuations [22, 23]. Violation of the Bell inequality requires mixing of the two outgoing channels at each end of the quantum dot (described by $2 \times 2$ unitary matrices $U_{L}$ and $U_{R}$ ). In order not to modify the degree of entanglement, this inter-channel scattering should be local, meaning that it should not lead to backscattering into the quantum dot. ${ }^{1}$ This might be done by making the barrier that separates lead L1 from L2 (and $\mathrm{R} 1$ from R2) partially transparent and tunable by a gate [23] (cf. Fig. 10.1, shaded rectangles). 


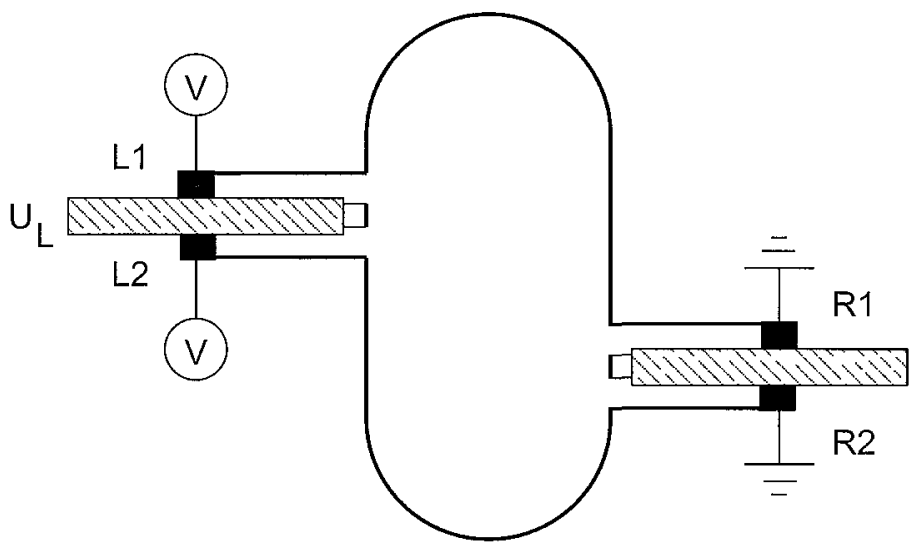

Figure 101 Sketch of the quantum dot entangles described in the text An electron leaving the quantum dot at the left or right represents a qubit, because it can be in one of two states' it is either in the upper channel $(\mathrm{L} 1, \mathrm{R} 1)$ or in the lower channel (L2,R2). An example of a maximally entangled Bell pai1 is the superposition $(|\mathrm{L} 1, \mathrm{R} 1\rangle+|\mathrm{L} 2, \mathrm{R} 2\rangle) / \sqrt{2}$.

\section{Relation between entanglement and transmission eigenvalues}

The incoming state,

$$
\left|\Psi_{1 \mathrm{n}}\right\rangle=\prod_{E_{\Gamma}<\varepsilon<E_{\Gamma}+e V} a_{L, 1}^{\dagger} a_{L, 2}^{\dagger}|0\rangle,
$$

factorizes into two occupied channels at the left and two empty channels at the right, so it is not entangled. Here $a_{L, \imath}^{\dagger}(\varepsilon)$ is the creation operator for an incoming excitation at energy $\varepsilon$ in channel $\imath$ at the left and $|0\rangle$ represents the Fermi sea at zero temperature (all states below $E_{F}$ filled, all states above $E_{F}$ empty). There is a corresponding set of creation operators $a_{R, 2}^{\dagger}$ at the right. We collect the creation operators in a vector $a^{\dagger}=\left(a_{L, 1}^{\dagger}, a_{L, 2}^{\dagger}, a_{R, 1}^{\dagger}, a_{R, 2}^{\dagger}\right)$. With this notation we can write the incoming state in the form

$$
\left|\Psi_{1 \mathrm{n}}\right\rangle=a^{\dagger} \cdot \sigma \cdot a^{\dagger}|0\rangle, \quad \sigma=\left(\begin{array}{cc}
(\imath / 2) \sigma_{y} & 0 \\
0 & 0
\end{array}\right)
$$

where the product over energies is implicit.

Multiple scattering in the quantum dot entangles the outgoing state in the two left channels with that in the two right channels. The vector of creation operators $b^{\dagger}$ for outgoing states is related to that of the incoming states by a unitary $4 \times 4$ scattering matrix: $b=S \cdot a \Leftrightarrow b^{\dagger} \cdot S=a^{\dagger}$. Therefore the outgoing state has the form

$$
\left|\Psi_{\text {out }}\right\rangle=b^{\dagger} \cdot S \cdot \sigma \cdot S^{T} \cdot b^{\dagger}|0\rangle .
$$


There are two methods to quantity the degree of entanglement of the outgoing state:

A. One can use the entanglement of formation $\mathcal{F}$ of the full state $\left|\Psi_{\text {out }}\right\rangle$. The entanglement of formation of a pure state is defined by [24]

$$
\mathcal{F}=-\operatorname{Tr}_{L} \rho_{L} \log \rho_{L}, \quad \rho_{L}=\operatorname{Tr}_{R}\left|\Psi_{\text {out }}\right\rangle\left\langle\Psi_{\text {out }}\right|,
$$

with $\operatorname{Tr}_{L}$ or $\operatorname{Tr}_{R}$ the trace over the degrees of freedom at the left or right. (The logarithm has base 2.) The entanglement of formation of the outgoing state is given in terms of the transmission eigenvalues by [6]

$$
\begin{aligned}
\mathcal{F}= & -(e V / h)\left[T_{1} \log T_{1}\left(1-T_{2}\right)+T_{2} \log T_{2}\left(1-T_{1}\right)\right. \\
& \left.+\left(1-T_{1}-T_{2}\right) \log \left(1-T_{1}\right)\left(1-T_{2}\right)\right] .
\end{aligned}
$$

For $T_{1}=T_{2}=1 / 2$ the rate of entanglement production is maximal, equal to $2 \mathrm{eV} / \mathrm{h}$ (bits per second).

B. Alternatively, one can project $\left|\Psi_{\text {out }}\right\rangle$ onto a state $\left|\Psi_{\text {out }}^{\prime}\right\rangle$ with a single excitation at the left and at the right, and use the concurrence $\mathcal{C}$ of this pair of qubits as the measure of entanglement. The (normalized) projected state is

$$
\left|\Psi_{\text {out }}^{\prime}\right\rangle=\frac{\left(1-n_{L, 1} n_{L, 2}\right)\left(1-n_{R, 1} n_{R, 2}\right)\left|\Psi_{\text {out }}\right\rangle}{\left\langle\Psi_{\text {out }}\left|\left(1-n_{L, 1} n_{L, 2}\right)\left(1-n_{R, 1} n_{R, 2}\right)\right| \Psi_{\text {out }}\right\rangle^{1 / 2}},
$$

with number operator $n_{X, \imath}=b_{X, \imath}^{\dagger} b_{X, \imath}$ (for $X=L, R$ ). The concurrence [25] is a dimensionless number between 0 (no entanglement) and 1 (a fully entangled Bell pair). ${ }^{2}$ The transmission formula for the concurrence is [6]

$$
\mathcal{C}=\frac{2\left[T_{1}\left(1-T_{1}\right) T_{2}\left(1-T_{2}\right)\right]^{1 / 2}}{T_{1}+T_{2}-2 T_{1} T_{2}}
$$

Full entanglement is reached when $T_{1}=T_{2}$, regardless of the value of the transmission.

Notice that in both methods A and B the degree of entanglement depends only on the transmission eigenvalues $T_{1}, T_{2}$, and not on the eigenvectors of the transmission matrix. Eqs. (10.6) and (10.7) hold irrespective of whether timereversal symmetry (TRS) is broken by a magnetic field or not. In Ref. [6] the expressions were simplified by specializing to the tunneling regime $T_{1}, T_{2} \ll 1$. Here we will not make this tunneling assumption.

In what follows we will concentrate on the concurrence $\mathcal{C}$ of the projected state $\left|\Psi_{\text {out }}^{\prime}\right\rangle$, since that is the quantity which is measured by correlating current fluctuations. The entanglement of formation $\mathcal{F}$ of the full state $\left|\Psi_{\text {out }}\right\rangle$ contains also contributions involving a different number of excitations at the left and at the right. Such contributions are not measurable with detectors that conserve particle number [26]. 


\section{Statistics of the concurrence}

The statistics of $\mathcal{C}$ is determined by the statistics of the transmission eigenvalues. For chaotic scattering their distribution is given by random-matrix theory [11],

$$
P\left(T_{1}, T_{2}\right)=c_{\beta}\left|T_{1}-T_{2}\right|^{\beta}\left(T_{1} T_{2}\right)^{-1+\beta / 2},
$$

with normalization constants $c_{1}=3 / 4, c_{2}=6$. We obtain the following values for the mean and variance of the concurrence in the case $\beta=1$ (preserved TRS) and $\beta=2$ (broken TRS):

$$
\begin{array}{r}
\langle\mathcal{C}\rangle= \begin{cases}0.3863 & \text { if } \beta=1, \\
0.3875 & \text { if } \beta=2,\end{cases} \\
\left\langle\mathcal{C}^{2}\right\rangle-\langle\mathcal{C}\rangle^{2}= \begin{cases}0.0782 & \text { if } \beta=1 \\
0.0565 & \text { if } \beta=2\end{cases}
\end{array}
$$

The effect of broken TRS on the average concurrence is unusually small, less than $1 \%$. In contrast, the conductance $G=\left(e^{2} / h\right) \operatorname{Tr} t t^{\dagger} \propto T_{1}+T_{2}$ increases by $25 \%$ upon breaking TRS. The main effect of breaking TRS is to reduce the sample-to-sample fluctuations in the concurrence, by about $15 \%$ in the root-mean-square value.

\section{Relation between Bell parameter and concurrence}

The Bell parameter $\mathcal{E}$ is defined by [22,23]

$$
\mathcal{E}=\max \left[E\left(U_{L}, U_{R}\right)+E\left(U_{L}^{\prime}, U_{R}\right)+E\left(U_{L}, U_{R}^{\prime}\right)-E\left(U_{L}^{\prime}, U_{R}^{\prime}\right)\right],
$$

where the maximization is over the $2 \times 2$ unitary matrices $U_{L}, U_{R}, U_{L}^{\prime}, U_{R}^{\prime}$ that mix the channels at the left and right end of the system. For given $U_{L}, U_{R}$ the correlator $E$ has the expression

$$
E=\frac{\left\langle\left(\delta I_{L, 1}-\delta I_{L, 2}\right)\left(\delta I_{R, 1}-\delta I_{R, 2}\right)\right\rangle}{\left\langle\left(\delta I_{L, 1}+\delta I_{L, 2}\right)\left(\delta I_{R, 1}+\delta I_{R, 2}\right)\right\rangle} .
$$

Here $\delta I_{L, i} \equiv I_{L, i}-\left\langle I_{L, i}\right\rangle$ is the low-frequency current fluctuation in the outgoing channel $i$ at the left ${ }^{3}$ and $\delta I_{R, j}$ is the same quantity for outgoing channel $j$ at the right. The average $\langle\cdots\rangle$ in this equation is over a long detection time for a fixed sample. (We will consider ensemble averages later.)

In the tunneling regime $T_{1}, T_{2} \ll 1$ there is a one-to-one relation $\mathcal{E}=$ $2 \sqrt{1+\mathcal{C}^{2}}$ between the Bell parameter $\mathcal{E}$ and the concurrence $\mathcal{C}$. Here we can not make the tunneling assumption. The Bell parameter (10.11) can then be larger than expected from the concurrence. The relation is [6]

$$
\begin{aligned}
\mathcal{E} & =2 \sqrt{1+\kappa^{2} \mathcal{C}^{2}}, \\
\kappa & =1+\frac{\left(T_{1}-T_{2}\right)^{2}}{T_{1}\left(1-T_{1}\right)+T_{2}\left(1-T_{2}\right)} .
\end{aligned}
$$


The amplification factor $\kappa \geq 1$ approaches unity if either $T_{1} \approx T_{2}$ or $T_{1}, T_{2} \ll$ 1.

Since $\mathcal{E}$ gives the amplified concurrence $\kappa \mathcal{C}$ rather than the bare concurrence $\mathcal{C}$, it is of interest to compare the moments of $\kappa \mathcal{C}$ with those of $\mathcal{C}$. By averaging with distribution (10.8) we find the mean and variance in a chaotic quantum dot:

$$
\begin{array}{r}
\langle\kappa \mathcal{C}\rangle= \begin{cases}0.7247 & \text { if } \beta=1, \\
0.8393 & \text { if } \beta=2,\end{cases} \\
\left\langle\kappa^{2} \mathcal{C}^{2}\right\rangle-\langle\kappa \mathcal{C}\rangle^{2}= \begin{cases}0.0838 & \text { if } \beta=1, \\
0.0393 & \text { if } \beta=2 .\end{cases}
\end{array}
$$

The amplification by $\kappa$ amounts to about a factor of two on average.

\section{Relation between noise correlator and concurrence}

For a different perspective on the relation between noise and entanglement, we write the correlator (10.12) of current fluctuations in a form that exposes the contribution from the concurrence.

Low-frequency correlators can be calculated with the help of the formula [27]

$$
\lim _{\omega, \omega^{\prime} \rightarrow 0}\left\langle\delta I_{L, i}(\omega) \delta I_{R, j}\left(\omega^{\prime}\right)\right\rangle=-\left(e^{3} V / h\right) 2 \pi \delta\left(\omega+\omega^{\prime}\right)\left|\left(r t^{\dagger}\right)_{i j}\right|^{2} .
$$

The reflection and transmission matrices $r, t$ are to be evaluated at the Fermi energy. We decompose these matrices in eigenvectors and eigenvalues,

$$
r=U_{L}\left(\begin{array}{cc}
\sqrt{1-T_{1}} & 0 \\
0 & \sqrt{1-T_{2}}
\end{array}\right) U_{0}, \quad t=U_{R}\left(\begin{array}{cc}
\sqrt{T_{1}} & 0 \\
0 & \sqrt{T_{2}}
\end{array}\right) U_{0}
$$

with $2 \times 2$ unitary matrices $U_{L}, U_{R}, U_{0}$. The matrix $r$ contains the reflection amplitudes from left to left and the matrix $t$ contains the transmission amplitudes from left to right. ${ }^{4}$

Substitution into Eq. (10.12) gives

$$
E=\left(1-2\left|U_{L, 11}\right|^{2}\right)\left(1-2\left|U_{R, 11}\right|^{2}\right)+4 \kappa \mathcal{C} \operatorname{Re} U_{L, 11} U_{R, 11}^{*} U_{L, 12}^{*} U_{R, 12} .
$$

We see that the entire dependence of the correlator $E$ on the transmission eigenvalues is through the product $\kappa \mathcal{C}$ of concurrence and amplification factor. This is the same quantity that enters in the Bell parameter (10.13). The correlator $E$ is less useful for the detection of entanglement than the Bell parameter $\mathcal{E}$, because it depends also on the matrices of eigenvectors $U_{L}, U_{R}$ - which the Bell parameter does not.

In a chaotic quantum dot the two matrices $U_{L}$ and $U_{R}$ are independently distributed in the circular unitary ensemble (a socalled "isotropic" distribution [11]). Averages over these matrices can be done conveniently in the 


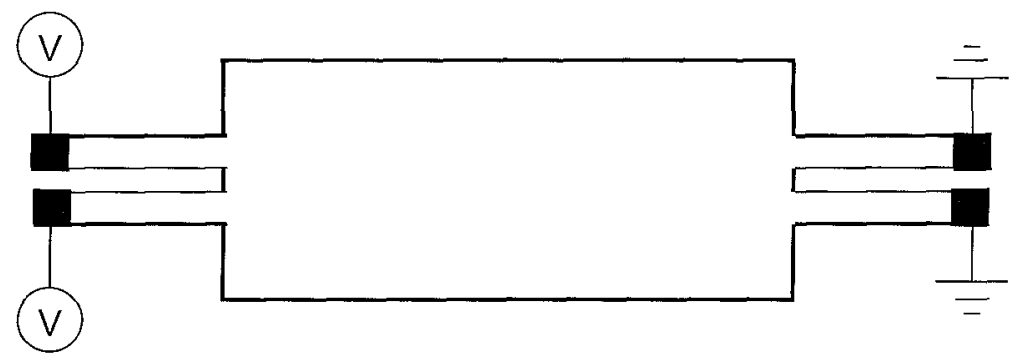

Figure 102 . The quantum dot of Fig. 10.1 has been replaced by a disordered wire (dotted rectangle). Although the distribution of transmission eigenvalues is different, the relation (10.23) between noise correlator and concurrence still applies. This relation only relies on the isotropy of the eigenvector distribution.

parametrization

$$
\begin{aligned}
& U=e^{\imath \alpha}\left(\begin{array}{cc}
e^{\imath \phi} \cos \gamma & e^{\imath \psi} \sin \gamma \\
-e^{-\imath \psi} \sin \gamma & e^{-\imath \phi} \cos \gamma
\end{array}\right), \\
& \gamma \in(0, \pi / 2), \quad \alpha, \phi, \psi \in(0,2 \pi) .
\end{aligned}
$$

The isotropic distribution implies that all four angles $\gamma, \alpha, \phi, \psi$ are independent. The distribution of $\alpha, \phi, \psi$ is uniform while the distribution of $\gamma$ is $P(\gamma) \propto$ $\sin 2 \gamma$.

With this parametrization Eq. (10.19) takes the form

$$
E=\cos 2 \gamma_{L} \cos 2 \gamma_{R}+\kappa \mathcal{C} \sin 2 \gamma_{L} \sin 2 \gamma_{R} \cos \left(\phi_{L}-\psi_{L}-\phi_{R}+\psi_{R}\right) \text {. }
$$

Upon averaging over the angles we find

$$
\langle E\rangle=0,\left\langle E^{2}\right\rangle=\frac{1}{9}+\frac{2}{9}\left\langle\kappa^{2} \mathcal{C}^{2}\right\rangle .
$$

The significance of this equation is that it applies generally to $2 \times 2$ transmission matrices with an isotropic distribution of eigenvectors, even if the distribution of eigenvalues differs from Eq. (10.8). For example, it applies to the disordered conductor shown in Fig. 10.2.

\section{Bell inequality without tunneling assumption}

The Bell parameter $\mathcal{E}$ is no longer directly related to the concurrence $\mathcal{C}$ if the transmission probabilities are not small compared to unity [6]: The relation (10.13) between $\mathcal{E}$ and $\mathcal{C}$ contains a spurious amplification factor $\kappa \geq 1$, which approaches unity in the tunneling regime. The same amplification factor appears in the correlator (10.19). In this section we show how one can avoid the amplification factor, by calculating the violation of the Bell inequality without making the tunneling assumption. The same problem was studied, from a different perspective, in Ref. [9]. 
As described in Ref. [22], the Bell inequality is formulated in terms of the correlator $K_{\imath \jmath}$ of the number of outgoing electrons detected in a time $\tau$ in channel $i$ at the left and channel $j$ at the right:

$$
\begin{aligned}
K_{\imath \jmath} & =\tau^{-2} \int_{0}^{\tau} d t \int_{0}^{\tau} d t^{\prime}\left\langle I_{L, \imath}(t) I_{R, \jmath}\left(t^{\prime}\right)\right\rangle \\
& =\left\langle I_{L, \imath}\right\rangle\left\langle I_{R, \jmath}\right\rangle+\int_{-\infty}^{\infty} d \omega \frac{2 \sin ^{2}(\omega \tau / 2)}{\pi(\omega \tau)^{2}} C_{\imath \jmath}(\omega) .
\end{aligned}
$$

Here $C_{\imath \jmath}(\omega)$ is the frequency dependent correlator of current fluctuations,

$$
C_{\imath \jmath}(\omega)=\int_{-\infty}^{\infty} d t e^{\imath \omega t}\left\langle\delta I_{L, \imath}(t) \delta I_{R, \jmath}(0)\right\rangle .
$$

In the tunneling limit it is possible to neglect the product of averages $\left\langle I_{L, \imath}\right\rangle\left\langle I_{R, y}\right\rangle$ and retain only the second term in Eq. (10.24), proportional to the current correlator $C_{\imath j}$. Both terms are needed if one is not in the tunneling limit.

We assume that $V$ is small enough that the energy dependence of the scattering matrix may be neglected in the range $\left(E_{F}, E_{F}+e V\right)$. (That requires $\mathrm{eV}$ small compared to the mean level spacing of the quantum dot.) Then the frequency dependence of $C_{\imath \jmath}(\omega)$ is given simply by ${ }^{5}$

$$
C_{\imath \jmath}(\omega)=C_{\imath \jmath}(0) \times\left\{\begin{array}{cl}
1-|\hbar \omega / e V| & \text { if }|\hbar \omega / e V|<1 \\
0 & \text { if }|\hbar \omega / e V|>1
\end{array}\right.
$$

For short detection times $\tau \ll h / e V$ one may take the limit

$$
\lim _{\tau \rightarrow 0} \int_{-\infty}^{\infty} d \omega \frac{2 \sin ^{2}(\omega \tau / 2)}{\pi(\omega \tau)^{2}} C_{\imath \jmath}(\omega)=\int_{-\infty}^{\infty} \frac{d \omega}{2 \pi} C_{\imath \jmath}(\omega)=\frac{e V}{h} C_{\imath \jmath}(0) .
$$

In view of Eq. (10.17), the zero-frequency limit of the current correlator $(10.25)$ is given by

$$
C_{\imath \jmath}(0)=-\left(e^{3} V / h\right)\left|\left(r t^{\dagger}\right)_{\imath \jmath}\right|^{2} .
$$

The mean outgoing currents are given by $\left\langle I_{L, \imath}\right\rangle=\left(e^{2} V / h\right)\left(r r^{\dagger}\right)_{\imath 2}$ and $\left\langle I_{R, \eta}\right\rangle=$ $\left(e^{2} V / h\right)\left(t t^{\dagger}\right)_{\jmath \jmath}$. Substitution into Eq. (10.24) gives the short-detection-time limit

$$
\begin{aligned}
\lim _{\tau \rightarrow 0} K_{\imath \jmath} & =\left\langle I_{L, \imath}\right\rangle\left\langle I_{R, \jmath}\right\rangle+(e V / h) C_{\imath \jmath}(0) \\
& =\left(e^{2} V / h\right)^{2}\left[\left(r r^{\dagger}\right)_{\imath \imath}\left(t t^{\dagger}\right)_{\jmath \jmath}-\left|\left(r t^{\dagger}\right)_{\imath \jmath}\right|^{2}\right] .
\end{aligned}
$$

We now define the correlator $\tilde{E}$ in terms of the short-time $K_{\imath \jmath}$,

$$
\tilde{E}=\frac{K_{11}+K_{22}-K_{12}-K_{21}}{K_{11}+K_{22}+K_{12}+K_{21}} .
$$


Notice that this definition of $\tilde{E}$ corresponds to definition (10.12) of $E$ if $K_{i j}$ is replaced by $C_{i j}(0)$. Substitution of Eq. (10.29) leads to

$$
\tilde{E}=-\left(1-2\left|U_{L, 11}\right|^{2}\right)\left(1-2\left|U_{R, 11}\right|^{2}\right)-4 \mathcal{C} \operatorname{Re} U_{L, 11} U_{R, 11}^{*} U_{L, 12}^{*} U_{R, 12},
$$

where we have used the parametrization (10.18). Apart from an overall minus sign, Eq. (10.31) is the same as Eq. (10.19) - but without the factor $\kappa$ multiplying the concurrence.

The maximal violation $\tilde{\mathcal{E}}$ of the Bell inequality is defined in the same way as in Eq. (10.11), with $E$ replaced by $\tilde{E}$. The result

$$
\tilde{\mathcal{E}}=2 \sqrt{1+\mathcal{C}^{2}}
$$

is the same as Eq. (10.13) - but now without the factor $\kappa$.

Since short-time detection experiments are very difficult in the solid state, the usefulness of Eq. (10.32) is that it allows one to determine the concurrence using only low-frequency measurements. It generalizes the result of Ref. [23] to systems that are not in the tunneling regime and solves a problem posed in Ref. [6] (footnote 24).

\section{Conclusion}

We have investigated theoretically the production and detection of entanglement by single-electron chaotic scattering. Much is similar to the tunneling regime studied earlier [6], but there are some interesting new aspects:

- The degree of entanglement, quantified by the concurrence $\mathcal{C}$, is sample specific. The sample-to-sample fluctuations become smaller if timereversal symmetry is broken, while the average concurrence is almost unchanged.

- The low-frequency current correlator $C_{i j}$ and the Bell parameter $\mathcal{E}$ constructed from it give the concurrence times an amplification factor $\kappa$. In the tunneling regime $\kappa \rightarrow 1$. One also has $\kappa=1$ if the two transmission eigenvalues $T_{1}, T_{2}$ are equal. The factor $\kappa$ can become arbitrarily large if $T_{1} \rightarrow 1$ and $T_{2} \rightarrow 0$ (or vice versa). On average, the amplification factor in an ensemble of chaotic quantum dots is about a factor of two.

- The bare concurrence, without the amplification factor, is obtained by adding to the low-frequency current correlator the product of average currents times $h / \mathrm{eV}$.

- The concurrence gives the maximal violation of the Bell inequality for detection times $\tau$ short compared to the coherence time $h / \mathrm{eV}$. In Ref. [22] the opposite limit $\tau \gg h / \mathrm{eV}$ was taken, which is appropriate in the 
tunneling regime, but does not allow to violate the Bell inequality outside of that regime. A similar conclusion was reached in Ref. [9].

From an experimental point of view, the missing building block in Fig. 10.1 is the local mixer at the left and right end of the quantum dot. These mixers are needed to isolate the contribution to the noise correlator that is due to the concurrence. In optics, a simple rotation of the polarizer suffices. The electronic analogue is a major challenge.

\section{Acknowledgments}

We have benefitted from comments on a draft of this manuscript by Markus Büttiker, Peter Samuelsson, and Eugene Sukhorukov. This research was supported by the Dutch Science Foundation NWO/FOM, by the U.S. Army Research Office (Grants DAAD 19-02-1-0086 \& DAAD-19-99-1-0215), and by the Harvard University CIMS Visitors Program.

\section{Notes}

1 The mixers have no effect on the incoming state, because both incoming channels are either filled or empty at any given energy

2 The concurrence $\mathcal{C}$ of the qubit pair is related to the entanglement of formation $\mathcal{F}^{\prime}$ of the projected state $\left|\Psi_{\text {out }}^{\prime}\right\rangle$ by $\mathcal{F}^{\prime}=-x \log x-(1-x) \log (1-x)$ with $x=\frac{1}{2}+\frac{1}{2} \sqrt{1-\mathcal{C}^{2}}$

3 The total current in channel $z$ at the left (incoming minus outgoing) is $e^{2} V / h-I_{L,}$,

4 In the presence of TRS one has $U_{0}=U_{L}^{S}$, but this constraint is 1rrelevant because anyway $U_{0}$ drops out of $\mathrm{Eq}(1017)$

5 The cross-correlator $C_{\imath \jmath}(\omega)$ vanishes for $|\hbar \omega|>\mathrm{eV}$ because we are correlating only the outgoing currents, the correlator of incoming plus outgoing currents contains also a voltage-independent term $\propto|\hbar \omega|$, cf Ref [28]

\section{References}

[1] L. Mandel and E. Wolf, Optical Coherence and Quantum Optics (Cambridge University, Cambridge, 1995).

[2] J. C. Egues, P. Recher, D. S. Saraga, V. N. Golovach, G. Burkard, E. V. Sukhorukov, and D. Loss, in Quantum Noise in Mesoscopic Physics, edited by Yu. V. Nazarov, NATO Science Series II. Vol. 97 (Kluwer, Dordrecht, 2003): pp. 241; T. Martin, A. Crepieux, and N. Chtchelkatchev, ibidem pp. 313.

[3] S. Scheel and D.-G. Welsch, Phys. Rev. A 64, 063811 (2001).

[4] M. S. Kim, W. Son, V. Bužek, and P. L. Knight, Phys. Rev. A 65, 032323 (2002).

[5] W. Xiang-bin, Phys. Rev. A 66, 024303 (2002).

[6] C. W. J. Beenakker, C. Emary, M. Kindermann, and J. L. van Velsen, Phys. Rev. Lett. 91, 147901 (2003). 
[7] L. Faoro, F. Taddei, and R. Fazio, cond-mat/0306733.

[8] C. W. J. Beenakker and M. Kindermann, cond-mat/0307103.

[9] P. Samuelsson, E. V. Sukhorukov, and M. Büttiker, cond-mat/0307473.

[10] G. B. Lesovik, A. V. Lebedev, and G. Blatter, cond-mat/0310020.

[11] C. W. J. Beenakker, Rev. Mod. Phys. 69, 731 (1997).

[12] L. P. Kouwenhoven, C. M. Marcus, P. L. McEuen, S. Tarucha, R. M. Westervelt, and N. S. Wingreen, in Mesoscopic Electron Transport, edited by L. L. Sohn, L. P. Kouwenhoven, and G. Schön, NATO ASI Series E345 (Kluwer, Dordrecht, 1997).

[13] Y. Alhassid, Rev. Mod. Phys. 72, 895 (2000).

[14] K. Furuya, M. C. Nemes, and G. Q. Pellegrino, Phys. Rev. Lett. 80, 5524 (1998).

[15] P. A. Miller and S. Sarkar, Phys. Rev. E 60, 1542 (1999).

[16] K. Zyczkowski and H.-J. Sommers, J. Phys. A 34, 7111 (2001).

[17] J. N. Bandyopadhyay and A. Lakshminarayan, Phys. Rev. Lett. 89, 060402 (2002).

[18] M. Žnidarič and T. Prosen, J. Phys. A 36, 2463 (2003).

[19] A. J. Scott and C. M. Caves, J. Phys. A 36, 9553 (2003).

[20] Ph. Jacquod, quant-ph/0308099.

[21] J. F. Clauser, M. A. Horne, A. Shimony, and R. A. Holt, Phys. Rev. Lett. 23, $880(1969)$.

[22] N. M. Chtchelkatchev, G. Blatter, G. B. Lesovik, and T. Martin, Phys. Rev. B 66, 161320(R) (2002).

[23] P. Samuelsson, E. V. Sukhorukov, and M. Büttiker, Phys. Rev. Lett. (to be published); cond-mat/0303531.

[24] M. A. Nielsen and I. L. Chuang, Quantum Computation and Quantum Information (Cambridge University, Cambridge, 2000).

[25] W. K. Wootters, Phys. Rev. Lett. 80, 2245 (1998).

[26] A. Peres, Phys. Rev. Lett. 74, 4571 (1995).

[27] M. Büttiker, Phys. Rev. Lett. 65, 2901 (1990).

[28] Ya. M. Blanter and M. Büttiker, Phys. Rep. 336, 1 (2000). 